Case Report

\title{
Rhabdomyolysis due to Trimethoprim-Sulfamethoxazole Administration following a Hematopoietic Stem Cell Transplant
}

\author{
Alexander Augustyn, ${ }^{1,2}$ Mona Lisa Alattar, ${ }^{2,3}$ and Harris Naina ${ }^{2,3}$ \\ ${ }^{1}$ Hamon Center for Therapeutic Oncology Research, University of Texas Southwestern Medical Center, Dallas, TX 75390, USA \\ ${ }^{2}$ Simmons Comprehensive Cancer Center, University of Texas Southwestern Medical Center, Dallas, TX 75390, USA \\ ${ }^{3}$ Department of Hematology and Oncology, University of Texas Southwestern Medical Center, Dallas, TX 75390, USA
}

Correspondence should be addressed to Harris Naina; harrisvk2002@yahoo.com

Received 30 July 2015; Revised 25 September 2015; Accepted 4 October 2015

Academic Editor: Marcel W. Bekkenk

\begin{abstract}
Copyright (C) 2015 Alexander Augustyn et al. This is an open access article distributed under the Creative Commons Attribution License, which permits unrestricted use, distribution, and reproduction in any medium, provided the original work is properly cited.

Rhabdomyolysis, a syndrome of muscle necrosis, is a life-threatening event. Here we describe the case of a patient with chronic myeloid leukemia who underwent a haploidentical stem cell transplant and subsequently developed rhabdomyolysis after beginning trimethoprim-sulfamethoxazole (TMP/SMX) prophylaxis therapy. Rechallenge with TMP/SMX resulted in a repeat episode of rhabdomyolysis and confirmed the association. Withdrawal of TMP/SMX led to sustained normalization of creatine kinase levels in the patient. A high index of suspicion is necessary to identify TMP/SMX as the cause of rhabdomyolysis in immunocompromised patients.
\end{abstract}

\section{Introduction}

Rhabdomyolysis is a potentially life-threatening syndrome of muscle necrosis characterized by the release of intracellular muscle contents into the systemic circulation and can result in significant muscle pain, electrolyte imbalance, acute renal failure, and even death $[1,2]$. Many medications, including salicylates, statins, neuroleptics, and fibrates, are associated with rhabdomyolysis although few reports indicate trimethoprim-sulfamethoxazole (TMP/SMX), a commonly used antibiotic, as the culprit [2-8]. Here we describe the case of a patient with blast phase chronic myeloid leukemia and subsequent haploidentical stem cell transplant maintained on dasatinib who developed rhabdomyolysis when concurrent TMP/SMX prophylaxis was initiated.

The classic triad of rhabdomyolysis includes muscle pain, weakness, and dark urine although the presentation can vary from asymptomatic elevations of muscle enzymes to severe muscle pain with acute kidney failure $[1,2]$. In addition to characteristic symptoms, about half of patients also present with myoglobinuria, while more severe cases can present with electrolyte imbalances such as hyperkalemia, acute renal failure, and/or swelling of the extremities $[9,10]$. The trademark laboratory diagnosis is an elevation of creatine phosphokinase (CK) to levels 5 times the normal limit, with a range of approximately 1,000 to 100,000 international units per liter (IU) [11].

The association of TMP/SMX with rhabdomyolysis is rare, and most cases have been reported in patients with human immunodeficiency virus (HIV) who receive TMP/ SMX as prophylaxis against Toxoplasma gondii and prophylaxis or treatment for Pneumocystis jirovecii pneumonia (PJP) $[3,4,6,7]$. TMP/SMX was also reported as the cause of rhabdomyolysis in one patient with CML who subsequently underwent an unrelated donor allogeneic stem cell transplant, developed PJP, and was treated with highdose TMP/SMX although without concurrent tyrosine kinase inhibitor (TKI) therapy [5]. A diagnosis of rhabdomyolysis was made after the patient developed lactic acidosis, acute renal failure, and hypotension with dramatic elevation of CK levels. Discontinuation of TMP/SMX led to CK normalization within five days [5]. Here, we report the case of a patient with CML and haploidentical stem cell transplant who developed rhabdomyolysis while receiving TMP/SMX for PJP prophylaxis. Discontinuation of all medications resulted in CK normalization while the rechallenge with TMP/SMX 
caused repeated elevation of CK levels, supporting the diagnosis.

\section{Case Presentation}

A 28-year-old male with a past medical history significant only for benign hypertension presented at our institution for swelling of the left mandible in 2011. Routine blood work revealed a white blood cell count (WBC) of 298,000 with $2 \%$ blasts. Peripheral blood polymerase chain reaction (PCR) was positive for the $t(9 ; 22)$ BCR-ABL translocation. The patient was started on imatinib after bone marrow biopsy confirmed the diagnosis of chronic myeloid leukemia, chronic phase (CML-CP). He initially achieved a complete hematologic response but six months later was found to have a WBC of 59,000 with $37 \%$ blasts and an elevated lactate dehydrogenase. Bone marrow biopsy revealed a mixed phenotype acute leukemia (B-cell/myeloid) most consistent with CML in blast phase. Due to progression on imatinib, he was treated with the R-hyper-CVAD regimen plus dasatinib while awaiting bone marrow transplantation.

Two years later, in January 2013, our patient received a haploidentical transplant and his course was free from graft versus host disease and major infections. He achieved major molecular response and was maintained on dasatinib. Six months after transplantation, his cytopenias resolved, immunosuppressive agents were tapered completely, and he was started on TMP/SMX and valacyclovir prophylaxis. Of note, the patient did not use any herbal remedies.

In September of 2013, the dasatinib dose was increased from $75 \mathrm{mg}$ daily to $100 \mathrm{mg}$ after tacrolimus was discontinued and he received five vaccinations (influenza, TDaP, HepB, Hib, and IPV). Four days later, our patient presented at his usual follow-up clinic visit with complaints of dark urine despite adequate water intake with no diarrhea or other symptoms. He did not report any abnormal exercise routines. Initial laboratory evaluation revealed LDH 3172 international units/L (IU/L), AST $1532 \mathrm{IU} / \mathrm{L}$, and ALT $321 \mathrm{IU} / \mathrm{L}$. The patient's baseline AST and ALT were $22 \mathrm{IU} / \mathrm{L}$ and $21 \mathrm{IU} / \mathrm{L}$, respectively, measured three months prior to admission. Immediately, all medications including dasatinib, TMP/SMX, amlodipine, valacyclovir, and pantoprazole were discontinued. CK was found to be markedly elevated at 132,400 IU/L. Fluids were administered and his CK dropped to 76,600 IU/L overnight; he was discharged one day later with $\mathrm{CK}$ at $43,700 \mathrm{IU} / \mathrm{L}$ along with instructions to avoid strenuous exercise and be followed up closely in the clinic. 11 days later, his CK levels normalized at 502 IU/L and the decision was made to restart dasatinib at $100 \mathrm{mg}$ per day. No other medications were restarted. Four days later, his CK was measured at $301 \mathrm{IU} / \mathrm{L}$, and PJP prophylaxis with TMP/SMX was restarted. One week later, the patient presented for a scheduled laboratory workup and was found to have a CK of 34,300 IU/L but was otherwise asymptomatic, with clear yellow urine.

The patient was admitted and TMP/SMX and dasatinib were once again held, fluids were administered, and his CK levels decreased to $8,300 \mathrm{IU} / \mathrm{L}$ when he was discharged two days later. Due to the temporal association of CK elevation

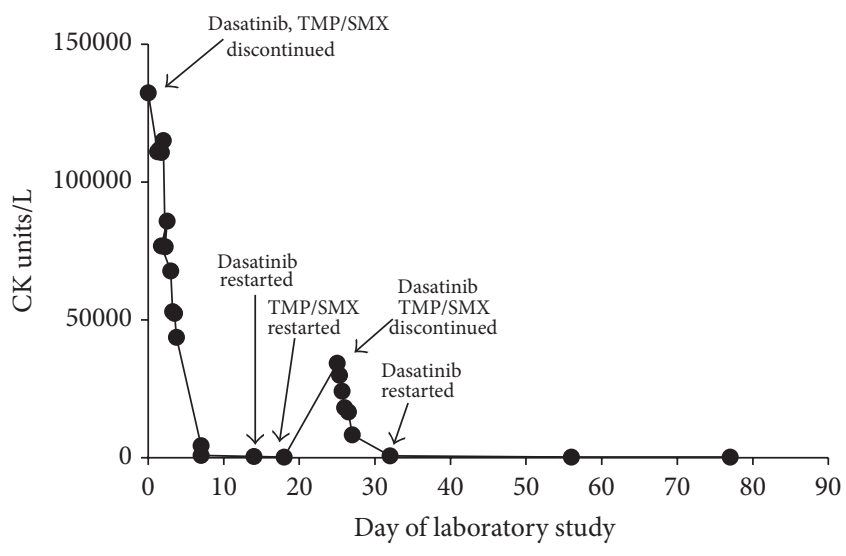

FIGURE 1: Plot of creatine kinase levels versus day of laboratory study. Relationship of creatine kinase levels to medication administration or cessation is indicated by arrows.

following rechallenge with TMP/SMX, the decision was made to not provide prophylaxis for PJP. The patient was continued on valacyclovir and dasatinib. Since TMP/SMX was completely stopped, his $\mathrm{CK}$ levels have remained normal (Figure 1). Complete medication dosing and CK, LDH, AST, and ALT levels for both inpatient hospitalizations are provided in Table 1.

\section{Discussion}

In a study involving 475 patients with rhabdomyolysis, exogenous toxins including medically administered drugs, alcohol, and illicit substances were determined to be the cause in $46 \%$ of cases [11]. Prior cases of TMP/SMX-induced rhabdomyolysis have occurred in patients with HIV receiving prophylaxis or treatment for toxoplasmosis or PJP $[3,4,6,7]$. One prior report detailed rhabdomyolysis in a patient with AML who underwent allogeneic stem cell transplant and developed PJP, necessitating treatment with TMP/SMX [5]. In our patient, before the discovery of TMP/SMX as the likely causative agent of rhabdomyolysis, we considered other etiologies, such as dasatinib, vaccination, or extreme exercise. Dasatinib use has been associated with rare occurrences of rhabdomyolysis ( $<1 \%$ of patients), according to the official drug data sheet, although no case reports currently detail such an association [12]. Vaccines for influenza and TDaP have also been temporally associated with the development of rhabdomyolysis in isolated case reports [13-15]. Based on the Naranjo probability scale of adverse drug reactions, TMP/SMX was the likely causative agent of rhabdomyolysis in our patient with a score of 6 (probable adverse drug reaction) [16]. This was confirmed by rechallenge with TMP/SMX, which resulted in elevation of CK to over 30,000 IU/L.

Several reports implicated imatinib as the cause of rhabdomyolysis. These patients were treated with imatinib for CML and aggressive fibromatosis [17-19]. In each case, withdrawal of imatinib or transition from imatinib to the secondgeneration tyrosine kinase inhibitor dasatinib resulted in 
TABLE 1: Complete medication and dosing history with relevant laboratory values for our patient during first inpatient admission for rhabdomyolysis (September 9, 2013), discharge (September 12, 2013), and outpatient clinic follow-up visit (September 27, 2013). The same information is also presented for the second inpatient admission for rhabdomyolysis (October 3, 2013), discharge (October 6, 2013), and outpatient clinic follow-up visit (October 14, 2013).

\begin{tabular}{|c|c|c|c|c|c|c|}
\hline & September 9 & September 12 & September 27 & October 3 & October 6 & October 14 \\
\hline \multicolumn{7}{|l|}{ Medications } \\
\hline Dasatinib & 100 mg daily & - & 100 mg daily & 100 mg daily & 100 mg daily & $100 \mathrm{mg}$ daily \\
\hline TMP/SMX & 160/800 MWF & - & 160/800 MWF & 160/800 MWF & - & - \\
\hline Valacyclovir & 500 mg daily & - & - & - & - & $500 \mathrm{mg}$ daily \\
\hline Amlodipine & 5 mg daily & - & 5 mg daily & 5 mg daily & 5 mg daily & 5 mg daily \\
\hline Pantoprazole & $40 \mathrm{mg}$ daily & - & $40 \mathrm{mg}$ daily & $40 \mathrm{mg}$ daily & 40 mg daily & $40 \mathrm{mg}$ daily \\
\hline \multicolumn{7}{|l|}{ Lab values (IU/L) } \\
\hline $\mathrm{CK}$ & 132,400 & 43,700 & 301 & 34,308 & 8,329 & 285 \\
\hline $\mathrm{LDH}$ & 3,172 & - & 203 & 647 & - & 210 \\
\hline AST & 1,532 & 978 & 26 & 535 & 198 & 26 \\
\hline ALT & 321 & 351 & 28 & 230 & 149 & 37 \\
\hline
\end{tabular}

resolution of rhabdomyolysis. Gordon et al. also identified a high number of $\mathrm{CK}$ abnormalities in patients treated with imatinib for CML or gastrointestinal stromal tumors, suggesting that this drug is associated with rare development of severe rhabdomyolysis [19]. However, to date, no report has directly linked dasatinib to rhabdomyolysis, and this remains true in the case of our patient whose CK levels have remained within normal limits on dasatinib maintenance therapy.

Drug-drug interactions such as those identified between cytochrome P450 isoform 3A4 inhibitors and HMG-CoA reductase inhibitors (statins) are known to cause rhabdomyolysis. For example, cotreatment with simvastatin and fluconazole, a known CYP isoenzyme 3A4 (CYP3A4) inhibitor, can cause rhabdomyolysis in patients likely due to elevated plasma levels of simvastatin [20]. Dasatinib is metabolized primarily by CYP3A4 and is a known time-dependent inhibitor of CYP3A4 [12, 21, 22]. TMP/SMX is a potent inhibitor of CYP2C8 and CYP2C9 and also inhibits CYP3A4 at higher concentrations [23]. However, the steady state plasma concentrations of both TMP (approximately $6 \mu \mathrm{M}$ ) and SMX (approximately $270 \mu \mathrm{M}$ ) are below that required to appreciably inhibit CYP3A4 in human cells (over $250 \mu \mathrm{M}$ for TMP, over $500 \mu \mathrm{M}$ for SMX, resp.), suggesting that a drug-drug interaction elevating levels of TMP/SMX and/or dasatinib leading to rhabdomyolysis is unlikely $[23,24]$. Of course, wide variability exists in cytochrome P450 enzymatic capacity among humans, so this possibility cannot be completely excluded at the present time [25]. The occurrence of drugdrug interactions increases as the number of medications increases and factors such as gastrointestinal absorption, drug distribution, and drug metabolism can enhance this effect [26]. Further study is needed to determine if a drug-drug interaction occurs between dasatinib and TMP/SMX, especially since both drugs are known to modulate CYP family members in vitro. If such an interaction is found to occur, pentamidine may be the preferred mode of PJP prophylaxis instead of TMP/SMX in the setting of concurrent TKI usage.

\section{Conflict of Interests}

The authors indicate no potential conflict of interests.

\section{Authors' Contribution}

Alexander Augustyn, Mona Lisa Alattar, and Harris Naina analyzed data, obtained funding, and wrote the paper.

\section{Acknowledgments}

Alexander Augustyn is supported by the UTSW Medical Scientist Training Program and the Ruth L. Kirschstein National Research Service Award for Individual Predoctoral MD/PhD Fellows (1F30CA168264).

\section{References}

[1] F. Y. Khan, "Rhabdomyolysis: a review of the literature," Netherlands Journal of Medicine, vol. 67, no. 9, pp. 272-283, 2009.

[2] R. Zutt, A. J. van der Kooi, G. E. Linthorst, R. J. A. Wanders, and M. de Visser, "Rhabdomyolysis: review of the literature," Neuromuscular Disorders, vol. 24, no. 8, pp. 651-659, 2014.

[3] S. Walker, J. Norwood, C. Thornton, and D. Schaberg, "Trimethoprim-sulfamethoxazole associated rhabdomyolysis in a patient with AIDS: case report and review of the literature," The American Journal of the Medical Sciences, vol. 331, no. 6, pp. 339341, 2006.

[4] S. J. Singer, J. A. Racoosin, and R. Viraraghavan, "Rhabdomyolysis in human immunodeficiency virus-positive patients taking trimethoprim-sulfamethoxazole," Clinical Infectious Diseases, vol. 26, no. 1, pp. 233-234, 1998.

[5] P. J. Kiel, N. Dickmeyer, and J. E. Schwartz, "Trimethoprimsulfamethoxazole-induced rhabdomyolysis in an allogeneic stem cell transplant patient," Transplant Infectious Disease, vol. 12, no. 5, pp. 451-454, 2010. 
[6] S. P. Jen and R. Sharma, "Trimethoprim-sulphamethoxazoleassociated rhabdomyolysis in an HIV-infected patient," International Journal of STD and AIDS, vol. 22, no. 7, pp. 411-412, 2011.

[7] H. J. Anders, J. R. Bogner, and F. D. Goebel, "Mild rhabdomyolysis after high-dose trimethoprim-sulfamethoxazole in a patient with HIV infection," European Journal of Medical Research, vol. 2, no. 5, pp. 198-200, 1997.

[8] B. Ainapurapu and U. B. Kanakadandi, "Trimethoprimsulfamethoxazole induced rhabdomyolysis," American Journal of Therapeutics, vol. 21, no. 3, pp. e78-e79, 2014.

[9] J. P. Knochel, "Rhabdomyolysis and myoglobinuria," Annual Review of Medicine, vol. 33, pp. 435-443, 1982.

[10] G. D. Giannoglou, Y. S. Chatzizisis, and G. Misirli, "The syndrome of rhabdomyolysis: pathophysiology and diagnosis," European Journal of Internal Medicine, vol. 18, no. 2, pp. 90-100, 2007.

[11] G. Melli, V. Chaudhry, and D. R. Cornblath, "Rhabdomyolysis: an evaluation of 475 hospitalized patients," Medicine, vol. 84, no. 6, pp. 377-385, 2005.

[12] Y. Fujii, M. Amano, and T. Seriu, "Pharmacological properties and clinical efficacy of dasatinib hydrate (Sprycel), an anticancer drug for chronic myelogenous leukemia and Philadelphia chromosome-positive acute lymphoblastic leukemia," Nihon Yakurigaku Zasshi, vol. 134, no. 3, pp. 159-167, 2009.

[13] K. S. Raman, T. Chandrasekar, R. S. Reeve, M. E. Roberts, and P. A. Kalra, "Influenza vaccine-induced rhabdomyolysis leading to acute renal transplant dysfunction," Nephrology Dialysis Transplantation, vol. 21, no. 2, pp. 530-531, 2006.

[14] H. Kulkarni, N. Lenzo, and A. McLean-Tooke, "Causality of rhabdomyolysis and combined tetanus, diphtheria and acellular pertussis (Tdap) vaccine administration," Journal of Clinical Pharmacology, vol. 53, no. 10, pp. 1099-1102, 2013.

[15] R. B. Callado, T. G. Ponte Carneiro, C. C. Da Cunha Parahyba, N. De Alcantara Lima, G. B. Da Silva Junior, and E. De Francesco Daher, "Rhabdomyolysis secondary to influenza A H1N1 vaccine resulting in acute kidney injury," Travel Medicine and Infectious Disease, vol. 11, no. 2, pp. 130-133, 2013.

[16] C. A. Naranjo, U. Busto, and E. M. Sellers, "A method for estimating the probability of adverse drug reactions," Clinical Pharmacology and Therapeutics, vol. 30, no. 2, pp. 239-245, 1981.

[17] N. Penel, J.-Y. Blay, and A. Adenis, "Imatinib as a possible cause of severe rhabdomyolysis," The New England Journal of Medicine, vol. 358, no. 25, pp. 2746-2747, 2008.

[18] U. Y. Malkan, G. Gunes, S. Etgul, T. Aslan, S. Balaban, and I. C. Haznedaroglu, "Management of de novo CML and imatinibinduced acute rhabdomyolysis with the second-generation TKI, dasatinib," Annals of Pharmacotherapy, vol. 49, no. 6, pp. 740742, 2015.

[19] J. K. Gordon, S. K. Magid, R. G. Maki, M. Fleisher, and E. Berman, "Elevations of creatine kinase in patients treated with imatinib mesylate (Gleevec)," Leukemia Research, vol. 34, no. 6, pp. 827-829, 2010.

[20] A. Shaukat, M. Benekli, G. D. Vladutiu, J. L. Slack, M. Wetzler, and M. R. Baer, "Simvastatin-fluconazole causing rhabdomyolysis," Annals of Pharmacotherapy, vol. 37, no. 7-8, pp. 1032-1035, 2003.

[21] A. M. Filppula, P. J. Neuvonen, and J. T. Backman, "In vitro assessment of time-dependent inhibitory effects on CYP2C8 and CYP3A activity by fourteen protein kinase inhibitors," Drug Metabolism and Disposition, vol. 42, no. 7, pp. 1202-1209, 2014.
[22] J. R. Kenny, S. Mukadam, C. Zhang et al., "Drug-drug interaction potential of marketed oncology drugs: in vitro assessment of time-dependent cytochrome $\mathrm{P} 450$ inhibition, reactive metabolite formation and drug-drug interaction prediction," Pharmaceutical Research, vol. 29, no. 7, pp. 1960-1976, 2012.

[23] X. Wen, J.-S. Wang, J. T. Backman, J. Laitila, and P. J. Neuvonen, "Trimethoprim and sulfamethoxazole are selective inhibitors of CYP2C8 and CYP2C9, respectively," Drug Metabolism and Disposition, vol. 30, no. 6, pp. 631-635, 2002.

[24] R. B. Patel and P. G. Welling, "Clinical pharmacokinetics of co-trimoxazole (trimethoprim-sulphamethoxazole)," Clinical Pharmacokinetics, vol. 5, no. 5, pp. 405-423, 1980.

[25] U. M. Zanger and M. Schwab, "Cytochrome P450 enzymes in drug metabolism: regulation of gene expression, enzyme activities, and impact of genetic variation," Pharmacology and Therapeutics, vol. 138, no. 1, pp. 103-141, 2013.

[26] C. Palleria, A. Di Paolo, C. Giofrè et al., "Pharmacokinetic drugdrug interaction and their implication in clinical management," Journal of Research in Medical Sciences, vol. 18, no. 7, pp. 601-610, 2013. 


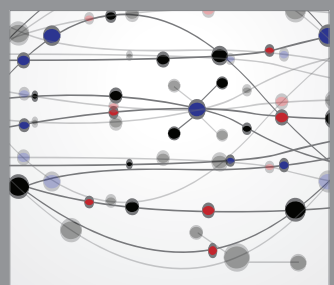

The Scientific World Journal
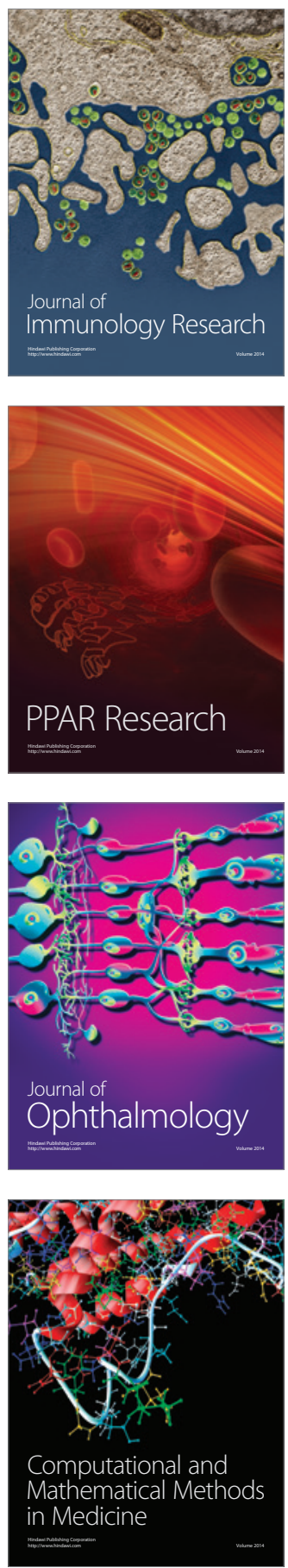

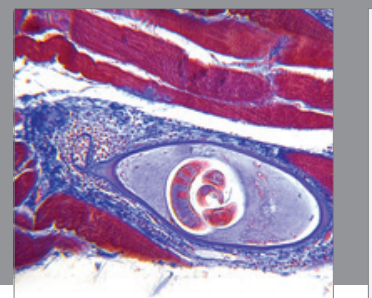

Gastroenterology

Research and Practice
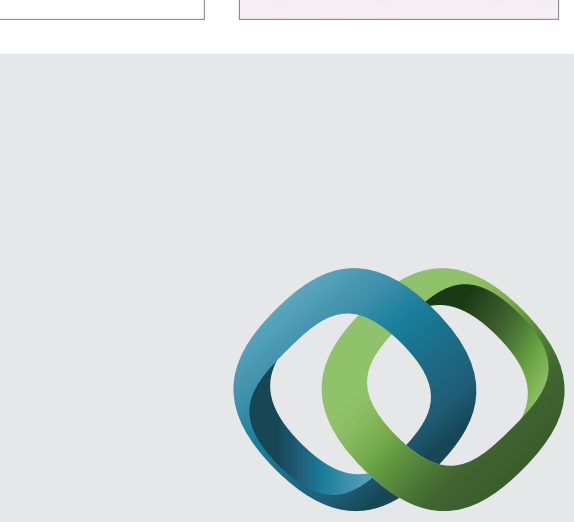

\section{Hindawi}

Submit your manuscripts at

http://www.hindawi.com
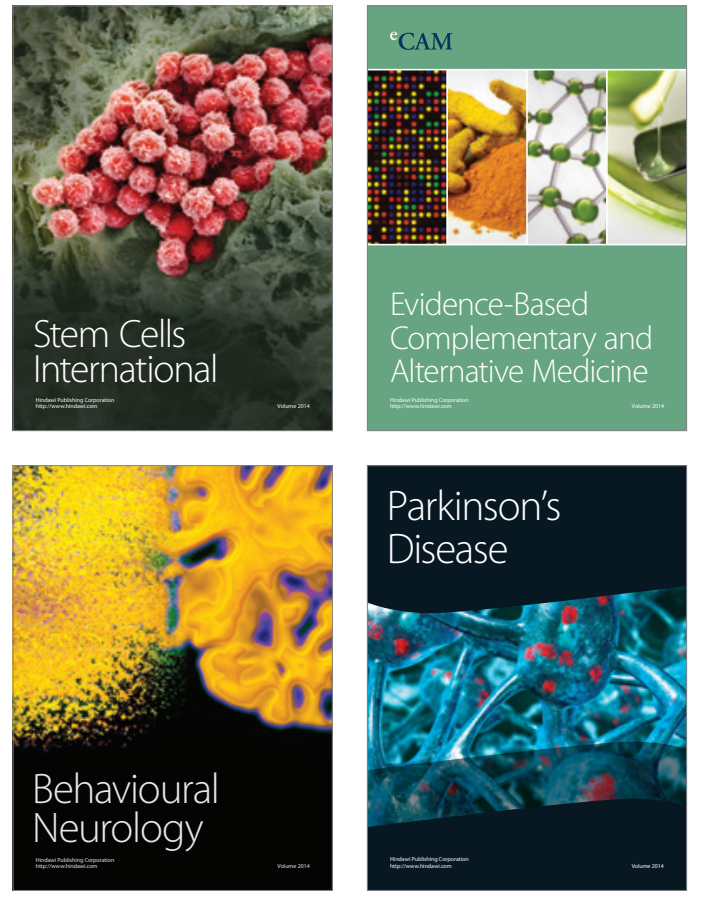
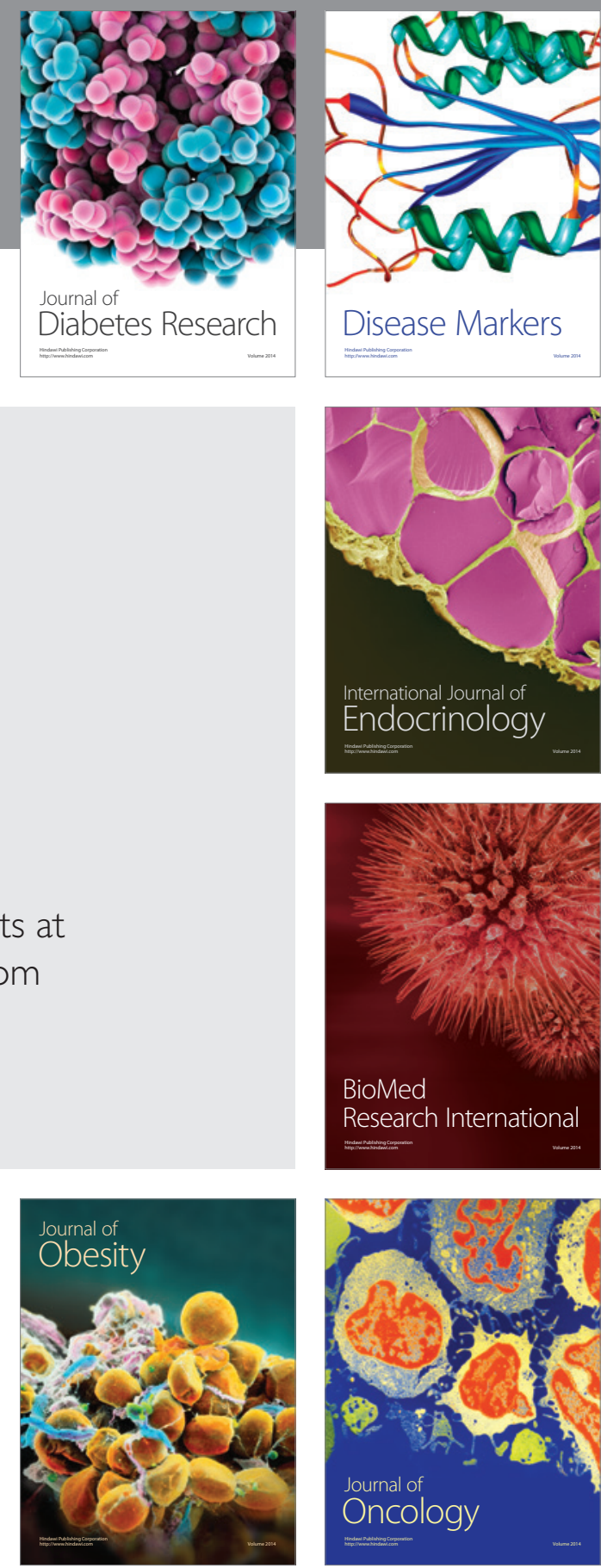

Disease Markers
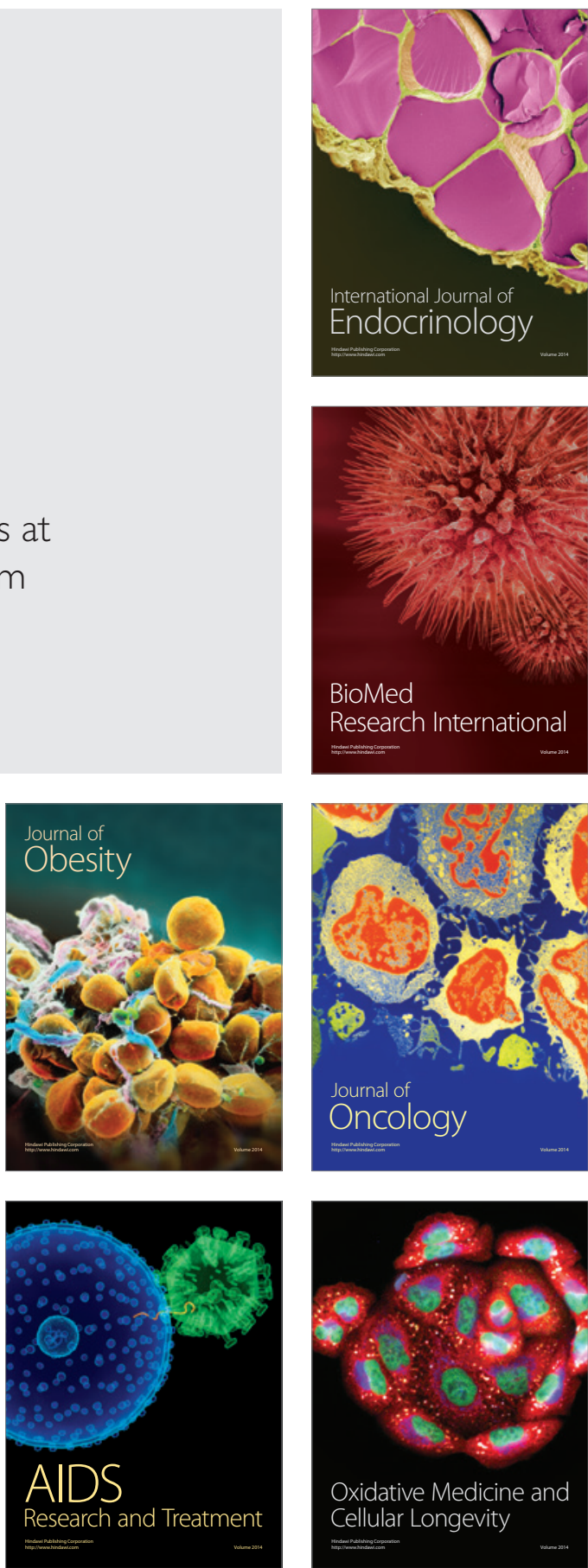